\title{
Search for neutrinoless double beta decay of ${ }^{76} \mathrm{Ge}$ with the GERmanium Detector Array "GERDA"
}

Riccardo Brugnera*†

Padova University, Italy

E-mail: brugnera@pd.infn.it

The study of neutrinoless double beta decay (DBD) is the only one presently known approach to the fundamental question if the neutrino is a Majorana particle, i.e. its own anti-particle. The observation of neutrinoless DBD would not only establish the Majorana nature of the neutrino but also represent a determination of its effective mass if the nuclear matrix element is given. So far, the most sensitive results have been obtained with ${ }^{76} \mathrm{Ge}$, and the group of Klapdor-Kleingrothaus has made a claim of discovery. Future experiments have to reduce radioactive backgrounds to increase the sensitivity. GERDA is a new DBD experiment which is currently under construction in the INFN Gran Sasso National Laboratory, Italy. It is implementing a new shielding concept by operating bare Ge diodes - enriched in ${ }^{76} \mathrm{Ge}$ - in high purity liquid argon supplemented by a water shield. The aim of GERDA is to verify or refute the recent claim of discovery, and, in a second phase, to achieve a two orders of magnitude lower background index than recent experiments, increasing the sensitive mass and reaching exposure of $100 \mathrm{~kg}$ yr. The paper will discuss design, physics reach, and status of construction of GERDA.

European Physical Society Europhysics Conference on High Energy Physics

July 16-22, 2009

Krakow, Poland

* Speaker.

${ }^{\dagger}$ On behalf of the GERDA Collaboration. 


\section{Introduction}

Since their discovery neutrinos have been an object of extensive experimental study and the knowledge about their properties has advanced our understanding of weak interactions significantly. Still unanswered, however, is the very fundamental question whether the neutrino is a Majorana particle like most extensions of the Standard Model assume. The study of DBD is the only one presently known approach to answer this question. If the decay occurs without the emission of neutrinos then their Majorana nature is proven. In the following, firstly we give a brief summary of the theory and the present experimental status; then in section 3 we describe the GERDA experiment: its goals and the detector; at the end its present status.

\section{Theory and present experimental programs}

Neutrinoless double beta decay, $\beta \beta(0 v)$, is a very slow lepton-number violating nuclear transition that happens if neutrinos have mass and are their own antiparticles. An initial nucleus $(Z, A)$ decays to $(Z+2, A)$, emitting two electrons. A related transition, called two-neutrino double DBD, $\beta \beta(2 v)$, results in the emission of two electron antineutrinos in addition to the electrons, also this is a process very rare but it does not violate any rule and in fact it has been observed in a number of experiments. If the $\beta \beta(0 v)$ decay is mediated by the exchange of a light Majorana neutrinos, the half-life is

$$
\left[T_{1 / 2}^{0 v}\left(0^{+} \rightarrow 0^{+}\right]^{-1}=G^{0 v}\left(Q_{\beta \beta}, Z\right)\left|M^{0 v}\right|^{2}\left\langle m_{\beta \beta}\right\rangle^{2}\right.
$$

where $G^{0 v}$ is the exactly calculable phase space integral, $M^{0 v}$ is the nuclear matrix element, $\left\langle m_{\beta \beta}\right\rangle$ is the effective Majorana mass of the electron neutrino:

$$
\left\langle m_{\beta \beta}\right\rangle \equiv\left|\sum_{k} m_{k} U_{e k}^{2}\right|
$$

Here the $m_{k}^{\prime} \mathrm{s}$ are the masses of the three light neutrinos and $U$ is the matrix that transforms states with well-defined mass into states with well-defined flavour. So the observation of neutrinoless DBD would not only establish the Majorana nature of the neutrino but also provide a measurement of its effective mass $m_{\beta \beta}$ and through the Eq. 2.2 the absolute mass scale for the neutrino can be investigated. In Eq. 2.1 the nuclear matrix element $M^{0 v}$ is not well known, presently most good calculations give the same result for a given matrix element to within a factor of 2 or 3 .

At present there is only one claim for a positive $\beta \beta(0 \mathrm{v})$ result by Klapdor et al. [1] as part of the Heidelberg-Moscow Collaboration: $T_{1 / 2}^{0 v}\left({ }^{76} \mathrm{Ge}\right)=2.23_{-0.31}^{+0.44} \times 10^{25} \mathrm{yr}$ at $1 \sigma$. The other most sensitive limits are from the IGEX experiment [2]: $T_{1 / 2}^{0 v}\left({ }^{76} \mathrm{Ge}\right) \geq 1.6 \times 10^{25} \mathrm{yr}(90 \%$ C.L.), and the CUORICINO experiment [3] : $T_{1 / 2}^{0 v}\left({ }^{130} \mathrm{Te}\right) \geq 3.0 \times 10^{24}$ yr (90\% C.L.). These experiments contained $5-10 \mathrm{~kg}$ of the parent isotope and ran for several years. Hence in order to increase the half-life sensitivity by a factor of about 100, the goal of the next generation of experiments will require hundreds of $\mathrm{kg}$ of parent isotope and a significant decrease in background beyond the present state of the art ( $\sim 0.1$ counts/(keV kg yr)). 


\section{The GERDA experiment}

The GERDA experiment [4 located in Hall A of the Laboratori Nazionali del Gran Sasso (LNGS) has the aim to search neutrinoless DBD using enriched ${ }^{76} \mathrm{Ge}$ detectors. The experiment will proceed in several phases. In phase I, existing enriched ${ }^{76} \mathrm{Ge}$ of Heidelberg-Moscow and IGEX experiments for a total mass of $18 \mathrm{~kg}$ will be used. The goal for the background index is $10^{-2}$ counts $/(\mathrm{keV} \mathrm{kg} \mathrm{yr})$. In these conditions after an exposure of $15 \mathrm{~kg} \mathrm{yr}$ it will be reached a $T_{1 / 2}^{0 v}>2.2 \times 10^{25} \mathrm{yr}$ checking the Klapdor's claim. In its phase II with additional $20 \mathrm{~kg}$ of enriched Ge detectors and a background of $10^{-3}$ counts/(keV kg yr) and a $100 \mathrm{~kg}$ yr exposure GERDA will reach the sensitivity of $T_{1 / 2}^{0 v}>2 \times 10^{26} \mathrm{yr}$ at $90 \%$ C.L. corresponding to about $130 \mathrm{meV}$ (with the Rodin et al. [5] matrix elements). Depending on the results obtained in phase I and phase II, it is also foreseen a phase III with the aim of reaching the $10 \mathrm{meV}$ scale, this requires $\mathscr{O}(1 \mathrm{t})$ of enriched germanium and represents another huge step, which can only be afforded in the context of a world-wide collaboration. GERDA, shown as an artist view in Fig. 1, will use naked Ge crystals immersed in liquid Ar. The shielding of external environmental backgrounds is realized using an onion-like structure consisting of, from outside to inside: hyper-pure water in a $10 \mathrm{~m}$ diameter tank shown open in Fig. 1, a stainless steal cryostat with internal lining of hyperpure copper and ultra-pure liquid Ar. The Ge diodes will be assembled into an array suspended in the centre of

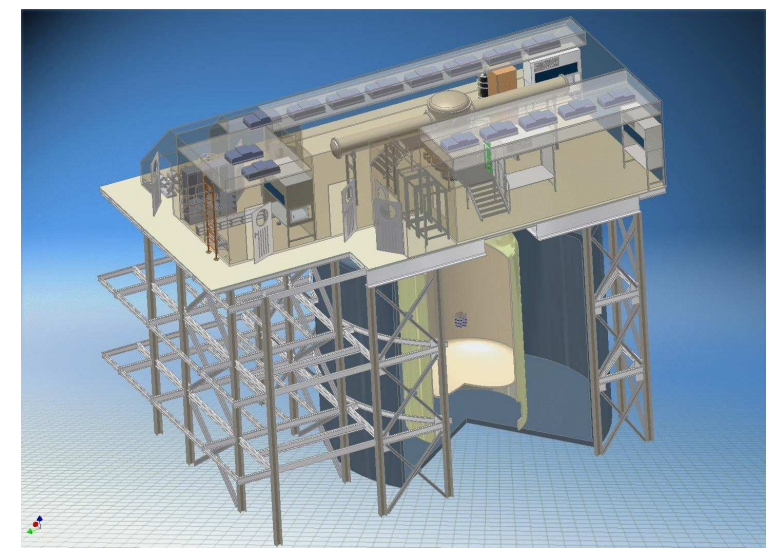

Figure 1: Artistic view of the GERDA experiment.

the liquid Ar volume. In Phase I the existing detectors are expected to contribute with about $10^{-2}$ counts/(keV kg yr), due to their intrinsic cosmogenic ${ }^{60} \mathrm{Co}$ contamination. This will dominate the background budget. However this will be enough to confirm or refute the Klapdor et al. evidence in an exposure of one year. Notice that this test needs to be performed on the same isotope, due to the uncertainty on the nuclear matrix elements. To reach the background index $10^{-3}$ counts/(keV $\mathrm{kg} \mathrm{yr}$ ), no individual component of the background budget should be larger than $10^{-4}$ counts/(keV $\mathrm{kg} \mathrm{yr}$ ). The method followed by GERDA to this aim is the following.

- The quantitative evaluation of all background sources in every component of the apparatus by analytical methods or Monte Carlo simulations. These calculations provide the allowed specific activity for each component, depending on its location relative to the detectors. 
- Shielding: $\gamma$ 's from the external environment (like $\left.{ }^{218} \mathrm{Tl}\right)$ with the $\mathrm{H}_{2} \mathrm{O}(3 \mathrm{~m}$ thickness) and the LAr ( $2 \mathrm{~m}$ thickness) shields; from the ${ }^{228} \mathrm{Th}$ in the stainless steel cryostat with a $\mathrm{Cu}$ shield and LAr; $\mu$-induced prompt signals with two vetoes. a plastic scintillator roof and the water used as Cherenkov medium.

- Mechanical design of minimum mass suspension and contact system for the Ge detectors. Notice also that in GERDA the detectors are surrounded by low $Z$ materials, a fact that minimizes the muon induced neutron background and makes the experiment feasible at the depth of Gran Sasso. Development of low mass ASIC and front-end electronics.

- Geometrical localization and pulse shape analysis to discriminate the backgrounds intrinsic to the Ge diodes. Indeed, the two electrons of the signal deposit their energy within a few millimeters, whilst the gammas, which constitute the principal background, do that with a few Compton scattering separated by a few centimeters. Consequently, the signal events are "single site" (SS); background events are "multiple site" (MS). SS can be distinguished from MS with pulse shape analysis and anti-coincidence between diodes.

The detectors for phase II are presently under development. Two different types are considered: a diode [6] with segmented electrodes ${ }^{1}$, and point-contact diode [7] developed as a commercial product by the company Canberra. Both detectors give similar suppression factors for the MS events and similar efficiency for SS events. The choice will be taken during the 2010.

\section{Outlook}

Presently (August 2009) all existing detectors made from enriched material have been tested to work properly and are ready to be installed. The water tank, the cryostat, the clean room have been already installed at LNGS. The final tests for the FE electronics and DAQ are well advanced. The commissioning phase will start this fall and the data-taking will follow immediately. In parallel intensive work is made in order to define the detectors for phase II.

\section{References}

[1] H.V. Klapdor-Kleingrothaus et al., Phys. Lett. B 586198 (2004); H.V. Klapdor-Kleingrothaus and I.V. Krivosheina, Mod. Phys. Lett. A 211547 (2006).

[2] IGEX Collab., C.E. Aalseth et al., Phys. Rev. D 65092007 (2002); IGEX Collab., C.E. Aalseth et al., Phys. Rev. D 70078302 (2004).

[3] CUORICINO Collab., C. Arnaboldi et al., Phys. Rev. C 78035502 (2008).

[4] GERDA Collab., I. Abt et al. GERDA The GERmanium Detector Array for the search of neutrinoless $\beta \beta$ decays ${ }^{76}$ Ge at $L N G S$ Proposal to the LNGS P38/04 September 2004.

[5] V.A. Rodin et al. Nucl. Phys. A 766107 (2006);

V.A. Rodin et al. Nucl. Phys. A 793213 (2007).

[6] I. Abt et al., N.I.M. A 577574 (2007).

[7] D. Dudjáš et al., IEEE Conference 2008, 19-25 October 2008, Dresden, Germany.

\footnotetext{
${ }^{1}$ The segmentation will be 6-fold in azimuth and three-fold in height.
} 